\title{
Wound Botulism in an Intravenous Drug User
}

\author{
Ashleigh Trimble* \\ Clinical Teaching Fellow, Crosshouse Hospital, Kilmarnock, Scotland \\ *Corresponding author: Ashleigh Trimble, Crosshouse Hospital, Kilmarnock, Scotland \\ Submission: 監October 14, 2017; Published: 眥 January 09, 2018
}

\begin{abstract}
Introduction: Wound botulism is a rare but potentially life-threatening neuroparalytic syndrome resulting from a neurotoxin elaborated by the bacterium Clostridium botilinum. No cases were reported in the UK prior to 2000, to date around 150 cases have been confirmed in injecting drug users.

Case presentation: In January 2015, an intravenous drug user presented with a 48-hour history of progressive blurred vision, diplopia, aphonia, dysphagia with evidence of type 2 respiratory failure. The patient could only communicate by writing and within two hours of hospital admission there was marked deterioration in condition with a progression of symmetrical descending paralysis necessitatinginvasive ventilation. Wound botulism secondary to infection with Clostridium botilinum producing toxin serotype B was confirmed.
\end{abstract}

Conclusion: There has been a cluster of cases of wound botulism in injecting heroin users in the West of Scotland. Recovery from the disease is slow with a high morbidity and mortality rate.

Keywords: Wound botulism; Intravenous drug user; Clostridium botilinum

\section{Introduction}

Cases of wound botulism are caused by the production of aneurotoxin by the gram-positive anaerobic bacterium Clostridium botulinum [1]. Seven serotypes of the disease have been found with type A, B, E and F causing human disease [1,2]. Cases of wound botulism are thought to be due to environmental contamination of injectable drugs, usually heroin, with bacterial spores $[1,3,4]$. The injectable drug has likely been diluted with a solvent, if some of the drug is injected intramuscularly or subcutaneously this increases the likelihood of tissue damage and botulism spores can germinate in these created anaerobic environments [1,3-5]. There were no reported cases of wound botulism in the UK prior to 2000, now there have been over 150 reports [1].

Between December 2014 and June 2015, Scotland had the largest outbreak of wound botulism recorded in Europe. A total of 40 cases were notified to Health Protection Scotland. All cases were intravenous drug users, 18 out of the 40 had microbiological confirmation of botulism diagnosis either by detection by bioassay or by isolation of Clostridium botulinum from debrided tissue by culture and PCR detection of neurotoxin genes. Otherwise the diagnosis of botulism was made clinically [5].

The incubation period can be as much as 2 weeks. Botulism toxin irreversibly disrupts stimulation-induced acetylcholine release at peripheral cholinergic synapses [1,3]. The resulting clinical syndrome is typically characterised by bilateral cranial nerve palsy and descending symmetrical muscle weakness $[1,3,6]$.

\section{Case Presentation}

In January 2015, A 32 year old female presented to a District General Hospital Emergency Department, her symptom timeline is as follows: three day history of diplopia and dysarthia, two day history of dysphagia and ataxia and one day history of dyspnoea and progression to only being able to write communications as verbally she felt 'too weak'.

She was cyanosed on arrival (oxygen saturations 81\%), with mild tachycardia $(100 \mathrm{bpm})$ with a raised respiratory rate. Her temperature was normal. Arterial blood gas showed acute type 2 respiratory failure. Lesions could be seen on her left forearm, right (dorsum) of hand and groins. There were no obvious clinical signs these lesions were infected. Mild crepitations were heard on respiratory examination but chest radiography showed no obvious infection.

The patient deteriorated rapidly in the department, within mere hours she now had a gasping respiratory effort, was aphonic with minimal facial movements and limited month opening. On examination, she had an absent gag reflex. It was known at this time she was an intravenous drug user who had last injected 5 days ago.

The patient was intubated in the Emergency Department, the Public Health Department was contacted given the high probability this could be another case of wound botulism and anti-toxin was transferred from the nearest available centre. The patient was also treated with broad-spectrum antibiotics to protect 
against secondary wound infections; literacy also supports this management plan point $[3,7,8]$.

Microbiology confirmed Clostridium botulinum type B from left forearm culture. The patient remained ventilated for 5 days and still had swallowing problems following extubation, the patient discharged against medical advice 3 days following this and died within 24 hours at home.

\section{Discussion}

From this outbreak, the mortality rate was $10 \%$ [5]. It is likely this patient died as a result of residual pharyngeal and respiratory muscle weakness which the body may not have been able to compensation for without supportive medical management.

The most common symptoms report from this outbreak was dysphagia and diplopia [5]. This patient presented to the Emergency Department with textbook symptoms associated with a neuromuscular blocking disease and the patient drug use made the diagnosis of Botulism and recent known outbreak made this the most likely differential diagnosis and allowed prompt management of the patient deteriorating clinical condition. Based on high probability of botulism, antiobiotics such as aminoglycosides were avoided due to their mechanism of action and prevent increased neuromuscular blockade which would have made symptoms more profound $[7,8]$.

Despite the unfortunate mortality outcome of this case, it showed the clinical importance of diagnosing such a disease early to allow prompt anti-toxin administration and respiratory supportive measures to increase survival chances.

\section{Acknowledgement}

I would like to thank Dr E McKenzie (EM consultant), Mr J Stevenson (EM consultant), Dr C McGuffie (EM consultant) and Dr N Perry (ICU Specialist Registrar) who all provided clinical expertise throughout this case and help to produce this case report.

\section{References}

1. Brett M, Hallas G, Mpamugo O (2004) Wound botulism in the UK and Ireland. J Med Microbiol 53(6): 555-561.

2. Dembek ZF, Smith LA, Rusnak JM (2007) Botulism: cause, effects, diagnosis, clinical and laboratory identification, and treatment modalities. Disaster Med Public Health Prep 2(2): 122-134.

3. Zhang JC, Sun L, Nie QH (2010) Botulism, where are we now? Clin Toxicol (Phila) 48(9): 867-879.

4. Gonzales y Tucker RD, Frazee B (2014) View from the front lines: an emergency medicine perspective on clostridial infections in injection drug users. Anaerobe 30: 108-115.

5. Martin SJ, Penrice G, Amar C, Grant K, Gorrie GH (2017) Wound botulism, its neurological manifestations, treatment and outcomes: A case series from the Glasgow outbreak, 2015. Scott Med J 62(4): 136-141.

6. Wenham T, Cohen A (2008) Botulism. Continuing Education in Anaesthesia Critical Care \& Pain 8(1): 21-22.

7. Santos JI, Swensen P, Glasgow LA (1981) Potentiation of Clostridium botulinum Toxin by Aminoglycoside Antibiotics: Clinical and Laboratory Observations. Pediatrics 68(1): 50-54.

8. Nigam PK, Nigam A (2010) Botulinum Toxin. Indian J Dermatol 55(1): 8-14. 